\title{
Sobre la valoración de la competencia matemática: claves para transitar hacia un enfoque interpretativo
}

\section{On the assessment of mathematical competence: keys in moving towards an interpretive approach}

\author{
Jesús Gallardo Romero, José Luis González Marí, Verónica Aurora Quintanilla Batallanos \\ Universidad de Málaga \\ gallardoromero@telefonica.net,gmari@uma.es, veronicaquintanilla@uma.es
}

RESUMEN: En este trabajo discutimos el problema de la interpretación de la competencia matemática en el aula. Con objeto de superar algunas de las controversias que afectan a la descripción por competencias del currículo de matemáticas, nos proponemos transitar hacia un enfoque interpretativo de la competencia matemática, en el que cobran un mayor protagonismo el conocimiento matemático y su comprensión. Mostramos la operatividad de nuestra propuesta a través de un ejemplo de interpretación de la competencia matemática de una alumna de secundaria involucrada en la resolución de una tarea algebraica específica. A partir de los usos dados al conocimiento matemático, se aportan evidencias de cómo emergen y se relacionan entre sí durante el mismo episodio de resolución rastros de comprensión y elementos de competencia matemática de diferente naturaleza.

PALABRAS CLAVE: competencia matemática; comprensión en matemáticas; interpretación; análisis epistemológico y fenomenológico; álgebra escolar.

ABSTRACT: In this paper we discuss the problem of the interpretation of mathematical competence in the classroom. In order to overcome some of the controversies affecting the approach by competencies, we intend to move towards an interpretative approach of mathematical competence in which the mathematical knowledge and their understanding are more significant. The potentiality of our proposal is shown through an example of interpretation of mathematical competence of a secondary student in solving a specific algebraic task. The uses of mathematical knowledge provide evidences on how the traces of mathematical competence and understanding of different nature emerge and they relate to each other.

KEYWORDS: mathematical competence; understanding in mathematics; interpretation; phenomenological and epistemological analysis; school algebra.

Fecha de recepción: abril 2013 • Aceptado: mayo 2014

Gallardo, J., González, J.L., Quintanilla, V.A. (2014) Sobre la valoración de la competencia matemática: claves para transitar hacia un enfoque interpretativo. Enseñanza de las 


\section{INTRODUCCIÓN}

En la actualidad, el desarrollo de la competencia matemática entre los estudiantes se presenta en el ámbito curricular como el objetivo prioritario de las matemáticas enseñadas en la educación básica (UNESCO, 2012). Se persigue fomentar la capacidad de los alumnos para resolver y responder adecuadamente en situaciones que requieren de la utilización del conocimiento matemático y de las destrezas propias del pensamiento matemático. El desarrollo del currículo basado en el fomento de las competencias pone el acento, sobre todo, en lo que los estudiantes saben hacer con las matemáticas, o más exactamente, en cómo pueden utilizar los conocimientos matemáticos aprendidos en la escuela para actuar en situaciones usuales de la vida cotidiana (Niss, 2002; Niss y Højgaard, 2011). Este enfoque aparece en estrecha relación con la perspectiva funcional de la matemática escolar, aquella en la que los conocimientos matemáticos se perciben esencialmente como herramientas con una clara finalidad y utilidad prácticas, cuyo uso permite resolver distintos problemas e interrogantes surgidos del entorno (Rico y Lupiáńez, 2008). Desde esta perspectiva se promueven las capacidades y destrezas de los alumnos en el aula para usar las matemáticas de una forma apropiada (con flexibilidad y eficiencia) en contextos y situaciones en los que pueden mostrarse pertinentes como medios de resolución (NTCM, 2000; OCDE, 2003). En la práctica, el desarrollo curricular de la competencia matemática exige transitar hacia sus componentes caracterizadores en forma de competencias específicas, elementos de la competencia matemática o capacidades concretas que buscan ser operativas. En este sentido, el enfoque por competencias comparte la intención de otras descripciones curriculares en las que también se plantean procedimientos y niveles de concreción, de lo general a lo específico, para garantizar su aplicabilidad. La novedad reside en que los descriptores particulares en los que se desgrana la competencia matemática en un ámbito de planificación más local se definen en términos de atributos externos prefijados que se utilizan para detallar la riqueza o potencialidad cognitiva del alumno. La competencia que subyace a la actividad matemática del estudiante queda entonces determinada por el dominio que este posee de los correspondientes atributos que la sustentan (Rico y Lupiáńez, 2008).

Si bien el desarrollo curricular por competencias está cada vez más extendido en educación matemática, recientemente han surgido diversas controversias desde el ámbito de la investigación que sugieren revisar sus planteamientos y matizar algunos de sus supuestos (Brown, 2008; Gresalfi, Martin, Hand y Greeno, 2009; Llewellyn, 2012; Puig, 2006). Las discusiones giran en torno a la vinculación de la competencia con el fenómeno de la comprensión en matemáticas, al estatus del conocimiento matemático como objeto de aprendizaje y a su protagonismo en relación con las situaciones problemáticas y las tareas matemáticas contextualizadas, o a la interpretación de la competencia matemática a partir de la actividad observable del estudiante, entre otras.

En este trabajo, con objeto de contribuir a la consolidación de la descripción del currículo por competencias, nos proponemos transitar hacia un enfoque interpretativo de la competencia matemática. Para ello, centramos la reflexión en el conocimiento matemático, en la comprensión y en la interpretación de la actividad matemática, e introducimos nuevos principios teórico-metodológicos que, siendo complementarios y compatibles con los del enfoque curricular, proporcionan en nuestra opinión respuestas satisfactorias a las distintas controversias planteadas. Nuestra propuesta se sustenta en un modelo operativo para la interpretación de la comprensión en matemáticas que venimos desarrollando en los últimos años (Gallardo y González, 2006; Gallardo, González y Quispe, 2008a, 2008b; Gallardo, González y Quintanilla, 2013, 2014).

Asimismo, evidenciamos la potencialidad práctica de nuestros planteamientos a través de un ejemplo de interpretación a nivel local de la competencia matemática de una alumna de $2 .^{\circ}$ de ESO involucrada en la resolución de una tarea algebraica específica. 


\section{CONTROVERSIAS EN TORNO A LA COMPETENCIA MATEMÁTICA DESDE UNA PERSPECTIVA CURRICULAR}

En esta sección delimitamos algunas cuestiones abiertas vinculadas a la dimensión normativa que se desprende de la descripción por competencias del currículo de matemáticas. Lo hacemos intentando profundizar en las relaciones de la competencia matemática con el fenómeno de la comprensión, con el propio conocimiento matemático, con las tareas y situaciones matemáticas y con la interpretación de la actividad matemática. Estas cuestiones afectan directamente a la naturaleza funcional de la competencia matemática así como a la operatividad de su valoración y desarrollo.

\section{Competencia y comprensión en matemáticas}

Para el enfoque curricular vigente, la competencia matemática plantea mayores requerimientos al alumno que la «simple» comprensión conceptual y procedimental de los objetos matemáticos, característica de discursos más tradicionales sobre la comprensión en matemáticas. De este modo, la comprensión conceptual, el conocimiento factual y la destreza procedimental se contemplan como prerrequisitos necesarios, aunque no suficientes, de la competencia matemática y se incluyen entre sus componentes fundamentales (NCTM, 2000: 21; Niss y Højgaard, 2011). Esta relación inclusiva es matizada, no obstante, por autores como Godino $(2002 a, 2002 b)$, quien sugiere un vínculo más bien complementario que inclusivo entre ambas funciones cognitivas. Una complementariedad entre el carácter funcional de las matemáticas vinculado a la competencia, por un lado, y la comprensión de las técnicas necesarias para realizar las tareas así como de las relaciones entre los diversos contenidos y procesos matemáticos puestos en juego, por otro.

Aunque puedan percibirse diferencias entre competencia y comprensión en la práctica de la enseñanza y el aprendizaje matemático, lo cierto es que las manifestaciones observables del uso del conocimiento matemático en diversos contextos, característico del alumno competente, solo constituyen una de las dimensiones que conforman el fenómeno complejo de la comprensión en matemáticas. De hecho, la preocupación fundamental por el desarrollo de la competencia matemática en los estudiantes forma parte de un problema más amplio en el que también intervienen otras dimensiones reconocidas de la comprensión tales como: origen y fuentes, naturaleza y funcionamiento, evolución, factores y efectos (Meel, 2003; Gallardo, González y Quintanilla, 2014). Y es precisamente en el carácter multidimensional de la comprensión donde radica una de las principales causas por la que su estudio resulta una tarea altamente compleja y un condicionante para las distintas propuestas encaminadas a interpretar, valorar y fomentar la competencia matemática. Estas iniciativas pueden verse afectadas por dificultades importantes en cuanto a su fundamentación y operatividad si no contemplan el desarrollo del aprendizaje y de la competencia matemática como un problema incluido en el de la comprensión en toda su extensión (Sierpinska, 2000). Si el marco de descripción del currículo por competencias no atiende en profundidad al fenómeno de la comprensión en matemáticas, tomando en consideración los avances sobre sus distintas dimensiones, pueden verse comprometidos la idoneidad del desarrollo de la competencia matemática y su valor como objetivo prioritario de la educación básica (Llewellyn, 2012).

\section{Competencia y conocimiento matemático}

El interés por el estudio de la complejidad fenomenológica y epistemológica de los conocimientos matemáticos y su consideración integral para la valoración y el desarrollo de la competencia podría ser más explícito y cobrar mayor protagonismo en el enfoque curricular actual. Más que en el apren- 
dizaje en sí de los conocimientos matemáticos en todas sus variantes básicas, el currículo basado en el fomento de la competencia matemática se organiza en torno a las capacidades y destrezas necesarias para afrontar con éxito tareas matemáticas particulares (Niss, 2002). Sin embargo, para Puig (2006) el currículo de matemáticas debería mantener la enseñanza de los conocimientos matemáticos pero como medios de organización de fenómenos. Más que enseñar los propios fenómenos en sí de forma directa a través de tareas específicamente diseñadas para ello, sugiere enseñar a los alumnos a organizar campos de fenómenos. Organizar fenómenos y campos de fenómenos no de cualquier forma, sino a través de un trabajo centrado en el análisis epistemológico y fenomenológico del conocimiento matemático. Las competencias se desarrollarían, precisamente, durante la realización de las actividades centradas en los aspectos conceptuales, estructurales y organizativos que surgen de los distintos análisis fenómenoepistemológicos de los conceptos matemáticos que organizan los fenómenos en estudio. Todo modelo de competencia y comprensión en matemáticas habría de involucrar un modelo del objeto que se va a estudiar que tuviera en cuenta las diversas facetas y estructuras del conocimiento matemático (Godino, 2002a). Cualquier análisis riguroso de los distintos elementos de la competencia matemática nos lleva a la adopción de un modelo epistemológico y fenomenológico sobre la propia matemática que responda, entre otras cuestiones, a cómo emergen los objetos matemáticos de las prácticas escolares (Font, Godino y Gallardo, 2013). Esto nos exige poner el énfasis tanto en la concepción de la naturaleza de las matemáticas que se está adoptando en la práctica docente, como en los fenómenos que organiza el conocimiento matemático y en las consecuencias que se derivan de ello (Puig, 2006).

\section{Interpretación de la competencia matemática}

En la práctica, el trazado metodológico hacia los componentes caracterizadores de la competencia matemática en forma de competencias específicas o capacidades concretas que buscan ser operativas, y definidas a su vez en términos de atributos externos, genera nuevos debates en el enfoque curricular, esta vez en torno a aspectos operativos relacionados con la interpretación de la competencia matemática.

En primer lugar, en la perspectiva curricular las competencias matemáticas específicas se refieren más bien a lo que los estudiantes necesitan dominar, conocer o saber hacer para ser considerados exitosos por el profesor o por sus compañeros en el aula de matemáticas. Se definen, por tanto, basándose en unas expectativas sobre lo que habría de considerarse como buenas prácticas matemáticas (prácticas correctas o aceptables), prefijadas desde el exterior y establecidas al margen de los acontecimientos que transcurren en cada aula particular. En contraposición a esta visión, autores como Brown (2008) buscan presentar la competencia matemática y la comprensión de los alumnos desde una perspectiva más inclusiva, en la que el objetivo es compartir diferentes modos de ver y comprender las matemáticas antes que insistir en una versión correcta de ellas y una supuesta buena comprensión o competencia. Un lugar alejado de la comprensión «estándar» establecida puede llegar a ser en ocasiones la situación cognitiva más deseable, confortable y segura para determinados alumnos (Llewellyn, 2012).

En segundo lugar, el carácter de atributo externo que el enfoque curricular asigna a la competencia matemática es discutible. Sobre este punto, Gresalfi, Martin, Hand y Greeno (2009) argumentan que las destrezas, habilidades o capacidades de referencia no deberían ser atribuidas externamente a los estudiantes porque en realidad son construidas en el interior de la clase de matemáticas y en contextos de participación conjunta con su profesor y compañeros. Estos autores muestran evidencias de los procesos de construcción de la competencia desde el interior de las clases de matemáticas.

Considerar la competencia como construida en interacción presta atención a las diferencias entre cómo podría ser definida la conducta competente en las aulas. Un estudiante considerado competente en un aula de matemáticas puede no ser necesariamente considerado competente en otra aula. Esto sugiere que las 
consideraciones acerca de quién es bueno en matemáticas deberían estar acompañadas por cuestiones sobre por qué las personas son buenas en matemáticas y, sobre todo, cómo lo sabemos (p. 52).

Este último aspecto hace referencia de nuevo a la necesidad de prestar una atención especial al fenómeno de la interpretación y valoración de la actividad matemática. Por otra parte, parece conveniente precisar más las características de las buenas prácticas en matemáticas, porque también se corre el riesgo de simplificar la complejidad inherente a la competencia matemática si en la práctica se mantienen caracterizaciones de sus atributos específicos en términos genéricos del tipo «plantear y resolver problemas» y similares (Puig, 2006).

En tercer lugar, establecer los atributos vinculados con la competencia matemática exige un ejercicio de inferencia que ha de realizarse a partir de las evidencias proporcionadas por las actuaciones externas del estudiante (acciones, comportamientos, decisiones, producciones escritas, etcétera) y las observaciones de su desempeño al enfrentar tareas matemáticas (Rico y Lupiańez, 2008). En este sentido, propuestas como la de Lupiáńez y Rico (2006) en el ámbito de la formación inicial de profesores permiten describir el modo en el que unas capacidades específicas relativas a un tema matemático particular contribuyen a la formación matemática general de los escolares en términos de competencias. En definitiva, el objetivo básico de desarrollar la competencia matemática de los escolares queda ligado de manera ineludible a la actividad de interpretar sus acciones matemáticas en el aula. Una circunstancia que nos permite situar de nuevo la interpretación en la base de las cuestiones abiertas fundamentales que atañen no solo a la perspectiva curricular de la competencia, sino al estudio de la competencia y la comprensión en matemáticas desde otros enfoques (Gallardo, González y Quintanilla, 2014).

\section{HACIA UN ENFOQUE INTERPRETATIVO DE LA COMPRENSIÓN Y LA COM- PETENCIA EN MATEMÁTICAS}

Con el propósito de contribuir al debate y superar algunas de las controversias suscitadas por la descripción por competencias del currículo de matemáticas, nos proponemos transitar hacia un enfoque más interpretativo de la competencia matemática en el que el conocimiento matemático, la comprensión y su interpretación cobran un mayor protagonismo. Los referentes que configuran nuestra visión incluyen una idea funcional de la comprensión compatible con la caracterización curricular de la competencia matemática. Asimismo, una propuesta de análisis epistemológico y fenomenológico del conocimiento matemático que posibilita la identificación y organización de tareas y la elaboración de instrumentos propicios para registrar la actividad matemática del estudiante. Esto aporta, no sin dificultades inherentes a la categorización y a la delimitación del universo de tareas para un conocimiento determinado, una primera referencia objetiva desde la que sustentar la interpretación. Por otra parte, también dirigimos la atención hacia el propio proceso de interpretación, incorporando un ciclo interpretativo que responde a la problemática transición entre tarea y actividad matemática, por un lado, y comprensión y competencia matemáticas, por otro. Con todo ello buscamos gestionar desde una posición más inclusiva la complejidad inherente a la interpretación de la competencia matemática, para de este modo contribuir a su valoración operativa desde la práctica docente.

\section{El conocimiento matemático como objeto de comprensión y referencia para la interpretación}

La interpretación de la actividad humana requiere de una intervención intencional por parte del intérprete que siempre se ve afectada por el problema abierto de la objetividad. En el ámbito de las ciencias sociales, el conocimiento generado por cualquier propuesta interpretativa está condicionado por la teoría con la que se interpreta (Popper, 2005). El intérprete participa e influye en el procedimiento 
de interpretación y las anticipaciones del sentido forman parte integrante del propio sentido que se va a interpretar (Habermas, 1999; Ricœur, 2002). Esta circunstancia no hay que entenderla, en todo caso, como una debilidad sino que forma parte de la propia naturaleza de la interpretación. El reto consiste en buscar referencias con las que poder garantizar un cierto grado de plausibilidad en los conocimientos adquiridos. La pertinencia de las propuestas interpretativas habría que buscarla no tanto en alguna supuesta veracidad adquirida sino en términos de su aplicabilidad o potencialidad práctica (Tahta, 1996).

Aspirando a ser operativa, nuestra propuesta interpretativa sitúa la referencia para la interpretación de la comprensión y competencia matemáticas en el propio conocimiento matemático y en sus usos dados durante la actividad matemática. Contemplamos el conocimiento matemático desde la perspectiva del alumno que aspira a comprenderlo. Como objeto de comprensión, el conocimiento matemático es considerado en nuestro enfoque como una entidad concreta de referencia con estructuras básicas específicas y exclusivas que delimitan su naturaleza y existencia. Estas estructuras surgen fundamentalmente de las relaciones con otros conocimientos matemáticos (estructura epistemológica) y de las situaciones y tareas problemáticas, campos y fenómenos que dan sentido al propio conocimiento (estructura fenomenológica). Ambas estructuras asociadas quedan constituidas de antemano para cada conocimiento matemático al margen del sujeto con pretensiones de comprensión. Los conocimientos matemáticos no siempre se utilizan del mismo modo y son los componentes caracterizadores de su estructura fenómeno-epistemológica los que establecen en cada caso los distintos requisitos condicionantes de su empleo intencionado por parte del individuo. Esta es la razón por la que proponemos tal estructura con fines interpretativos como referencia objetiva con la que certificar y caracterizar los usos dados al conocimiento en la actividad matemática.

\section{La competencia matemática como consecuencia de la comprensión del conocimiento matemático}

Por nuestra parte, consideramos que toda actividad matemática está propiciada por, y es consecuencia de, una actividad intelectual que demanda unas exigencias cognitivas necesariamente vinculadas a la esfera mental de quien la desarrolla. En este contexto situamos la comprensión en matemáticas, concibiéndola como fenómeno mental de carácter cognitivo, como actividad intelectual cognitiva específica, que capacita o hace competente al individuo para elaborar respuestas adaptadas y contextualizadas (observables o no) que involucran la utilización (registrable e interpretable, en su caso) del conocimiento matemático en alguna de las categorías y formas posibles de su dimensión fenómeno-epistemológica. Así es cómo relacionamos la competencia matemática con el punto de vista funcional de la comprensión a través del uso del conocimiento matemático. Ser competente en matemáticas es consecuencia de la comprensión del conocimiento matemático en el sentido que aquí presentamos. Desde el punto de vista de su formación o constitución, la comprensión de un conocimiento matemático está ligada a las experiencias matemáticas que se producen a través de las situaciones en las que interviene dicho conocimiento. En este sentido, desde el punto de vista de la observación, interpretación y valoración, los estudiantes manifiestan una cierta comprensión en relación con un conocimiento matemático concreto cuando, ante situaciones de desequilibrio cognitivo que deciden voluntariamente abordar, elaboran y emiten a su satisfacción respuestas adaptadas en las que hacen un uso significativo (esto es, libre, consciente e intencional) de este conocimiento. Este uso por parte del alumno, como forma de acción observable e interpretable, da cuenta de su comprensión. Por ello admitimos que un estudiante comprende un conocimiento matemático si es capaz de emplearlo, de manera eficaz en alguna de sus formas posibles, en todas aquellas situaciones pertenecientes a su ámbito fenómeno-epistemológico. Por esta razón, lo que un alumno utiliza y cómo lo utiliza para elaborar y emitir voluntariamente una 
respuesta adaptada a una situación, proporciona información específica sobre lo que comprende y cómo lo comprende. Según sea dicha utilización en cuanto a disponibilidad, diversidad y efectividad, así será la comprensión manifestada en el momento de responder. También cabe la posibilidad de una mayor comprensión que la revelada a través de una acción observable, pero nunca menor si la implicación es importante y voluntariamente decidida y el rendimiento es alto en la elaboración y valoración de la respuesta.

\section{El conocimiento matemático como organizador de tareas y experiencias matemáticas}

Nuestra visión sobre la comprensión y la competencia matemática requiere procedimientos para la identificación y selección de aquellas tareas problemáticas para plantear a los estudiantes, vinculadas al conocimiento matemático y generadoras de experiencias matemáticas observables. La estrategia sugerida por nuestro enfoque radica en determinar situaciones representativas de la parte de la estructura fenómeno-epistemológica del conocimiento matemático cuya comprensión se desea valorar, y con potencialidad para reflejar tal estructura a nivel cognitivo a través de los comportamientos y las respuestas externas de los alumnos, ambas condiciones necesarias para garantizar la utilidad de esas situaciones como instrumento operativo en la valoración de la comprensión. En ello se manifiesta un claro interés por profundizar en la complejidad del conocimiento matemático y por establecer dimensiones, categorías o componentes con los que controlar dicha complejidad de manera efectiva y operativa.

$\mathrm{Al}$ igual que ocurre con otros campos de conocimiento, el sujeto accede y comprende un conocimiento matemático a través de las experiencias significativas en situaciones en las que dicho conocimiento interviene de manera destacada; los usos que el sujeto da al conocimiento matemático como consecuencia de un proceso educativo no dependen solo de las estructuras y propiedades previas del conocimiento, sino de las experiencias del sujeto con dicho conocimiento, las cuales, en toda su riqueza, exceden normalmente de las limitadas experiencias matemáticas que se pueden proporcionar dentro de un aula o en el seno de una institución; todas ellas, experiencias internas y externas al aula, de matemáticas puras o aplicadas, en situaciones realistas o cotidianas o inventadas o formales, configuran la comprensión del conocimiento involucrado y contribuyen al desarrollo de la competencia matemática. Todas son experiencias en las que el conocimiento matemático funciona como conjunto de medios para disminuir la incertidumbre estructural, genérica o modélica ligada a las situaciones en las que actúa. El sujeto que participa activamente en estas experiencias significativas construye, recuerda, incorpora, reorganiza, completa, comprende y mejora sus posibilidades de actuación, es decir, mejora su competencia matemática.

\section{La comprensión matemática interpretada en términos de usos dados al conocimiento matemático}

Nuestra propuesta interpretativa comienza a nivel cognitivo con el reconocimiento de la comprensión matemática como fenómeno mental, irrumpe en el ámbito semiótico con el análisis de la actividad matemática del estudiante y desemboca en una superación fenómeno-epistemológica que nos permite retornar de nuevo a la comprensión del alumno a través de los usos dados al conocimiento matemático.

En primer lugar, asumimos que los fenómenos cognitivos no son radicalmente incomunicables. La comprensión matemática se constituye y exterioriza a través del lenguaje en sus distintas manifestaciones (Duval, 2006). Basándonos en esto, el registro observable generado durante el quehacer matemático se erige como la principal fuente depositaria de las expresiones o los rastros visibles derivados de la comprensión. En definitiva, la comprensión es comunicable e incluye en su manifestación externa rastros interpretables. 
En segundo lugar, la interpretación demanda el registro escrito de todo lo observable. La estabilidad, perdurabilidad e independencia del registro escrito en sus distintas manifestaciones lo hacen especialmente idóneo para desplegar en él cualquier esfuerzo interpretativo. Por otra parte, la inscripción de lo observable hace patente el progresivo distanciamiento entre lo mental, lo verbal, lo realizado y, finalmente, lo escrito, poniéndose de manifiesto la inaccesibilidad directa de los aspectos internos de la comprensión, la imposibilidad de una relación especular entre lo verbal y lo escrito y la exigencia ineludible de una interpretación dirigida al texto.

En tercer lugar, la interpretación persigue identificar los rastros de comprensión diseminados en el registro escrito y caracterizar a partir de ellos los usos del conocimiento matemático. Aunque la comprensión y la interpretación se ejerzan sobre la mediación de un texto, rebasan el campo de lo meramente semiótico:

Si la producción de cualquier expresión matemática puede ser vista como una acción, el significado de esa expresión está sujeto necesariamente a una interpretación que trasciende cualquier significado en la propia expresión. Es necesario buscar la forma en que la expresión está siendo utilizada por el individuo en un contexto particular. [...] El significado de cualquier acción matemática va más allá de lo que se encontraría en una investigación puramente literal o simbólica (Brown, 2001: 26).

El hecho de que la capacidad para utilizar el conocimiento matemático dependa en buena medida de su comprensión, nos obliga a situar la referencia última de la comprensión del estudiante, no ya en el registro escrito, sino en el uso del conocimiento matemático que deja entrever. Esto es, bajo la premisa de no poderse aplicar aquello que no se posee, encauzamos la búsqueda de la comprensión matemática en una dirección que parte de un texto pero prosigue más allá de él, hacia el empleo del conocimiento matemático. La interpretación se dirige entonces a la exteriorización y caracterización de los usos del conocimiento matemático que se desprenden de los rastros de comprensión emergentes de los registros escritos.

\section{Pautas para una interpretación operativa de la comprensión y competencia en matemáticas}

En la práctica, el agente intérprete debe enfrentar distintos requerimientos metodológicos a la hora de aplicar el procedimiento para la interpretación de la comprensión y competencia que se desprende de nuestro enfoque. De manera específica sugerimos las siguientes acciones que habría que considerar: (a) realización de un análisis epistemológico y fenomenológico del conocimiento matemático; (b) identificación de los elementos fenómeno-epistemológicos de este influyentes a nivel cognitivo, que son los responsables, entre otros aspectos, de la caracterización de los alumnos en términos de comprensión; (c) organización y selección de las situaciones y tareas matemáticas que dan sentido al conocimiento matemático, basándose en el resultado de los análisis previos; (d) garantía de que los estudiantes se enfrenten a situaciones pertenecientes a las distintas categorías surgidas del cruce de las estructuras epistemológica y fenomenológica del conocimiento matemático; $(e)$ configuración de los escenarios básicos hacia los que dirigir la interpretación; $(f)$ interpretación de la comprensión de los escolares en términos de capacidad para enfrentar con éxito las situaciones planteadas; $(g)$ identificación y validación de los rastros de comprensión matemática diseminados a lo largo del registro escrito, y $(b)$ revelación en estos rastros de los usos dados al conocimiento matemático empleando la propia estructura fenómeno-epistemológica del conocimiento como referencia objetiva con la que certificar tales usos. 


\section{INTERPRETANDO LA COMPETENCIA MATEMÁTICA EN LA PRÁCTICA: UN EJEMPLO ILUSTRATIVO}

La interacción en el aula y la toma de conciencia de la actividad matemática por parte de los estudiantes configuran un escenario de interpretaciones de la comprensión y la competencia matemáticas mediadas por el contexto de la tarea y por el contexto social y cultural de los estudiantes (Brown, 1996). De una forma compatible con la visión adoptada por la descripción por competencias del currículo de matemáticas, queremos mostrar que es posible ampliar el campo de acción de la competencia matemática frente a las tareas y situaciones problemáticas, así como afrontar su valoración en la práctica de un modo operativo ante tales tareas. En esta sección mostramos con un ejemplo la potencialidad práctica de nuestra propuesta interpretativa para la comprensión y la competencia matemáticas.

\section{Escenario de interpretación y tarea matemática}

La interpretación de la actividad matemática transcurre en diferentes escenarios básicos que se originan en el aula cuando el profesor pretende obtener información sobre la comprensión y competencia de los alumnos en distintas fases del proceso de enseñanza-aprendizaje. Por sus particularidades, conformadas por los elementos y las relaciones específicas que intervienen en ellos, resulta aconsejable concretar para cada escenario una caracterización particular que incluya una propuesta propia de gestión de la interpretación en él. En nuestro caso, nos interesa desplegar nuestro enfoque interpretativo en escenarios que compartan la complejidad propia de las situaciones hermenéuticas condicionadas por el lenguaje y en los que la implicación de los estudiantes sea importante, prolongada y con rendimiento alto en la elaboración de las respuestas. Escenarios en los que la actividad del alumno transcurra de un modo natural en el interior de sus clases de matemáticas cotidianas y al margen de condicionantes externos. Este es el caso del escenario que tomamos en esta ocasión como ejemplo para su interpretación. Se trata del protocolo de resolución de una tarea algebraica elaborado y redactado por Nadia, alumna de $2 .^{\circ}$ de ESO (13 ańos) en febrero de 2012. El desarrollo de protocolos como este formaba parte de la actividad matemática que Nadia desempeñaba con regularidad en su aula. Al ser una alumna acostumbrada a este tipo de trabajo matemático, se pudo garantizar una implicación seria y una participación decidida en la resolución de la tarea. Asimismo, al estar interesados en evidenciar la aplicabilidad de nuestra propuesta, en este episodio nos hemos preocupado por profundizar en la interpretación de las acciones matemáticas desplegadas por Nadia más que por buscar algún tipo de representatividad en lo realizado por ella.

Por su parte, la tarea propuesta, elegida entre las actividades incluidas en su libro de texto para el estudio de los polinomios, fue la siguiente:

Elige dos números de: $1,2,3,4,5$ y 6 , y colócalos en los triángulos para que la expresión: $5 \cdot(2 x-\Delta)-(\nabla x+3) \cdot(x-2)$ tome el valor 0 cuando $x=1$ (Álvarez, Hernández, Miranda, Moreno, Parra, Redondo, Redondo et al., 2008: 109).

Se trata de un problema de matemáticas cuya resolución exige el uso, conjunto y relacionado, de distintos conocimientos matemáticos específicos propios de los ámbitos aritmético y algebraico, así como el empleo de diversas estrategias de gestión y control del proceso de resolución propias del quehacer matemático. Por tanto, es una tarea que forma parte de la estructura fenómeno-epistemológica de varios conocimientos matemáticos. La hemos seleccionado porque esta complejidad favorece el establecimiento de vínculos situación-conocimiento. Aspiramos a mostrar la potencialidad de nuestra propuesta interpretativa para identificar y analizar estos vínculos fenómeno-epistemológicos. Qué vínculos establece la alumna y de qué manera lo hace entre las situaciones que van apareciendo durante 
la resolución y los posibles conocimientos matemáticos que pueden ir empleándose como medios para solventarlas. Ejemplos de ello serían, entre otros, decidir evaluar la expresión algebraica para un valor dado o manipular de un modo flexible condiciones iniciales para la incógnita. De este modo, van emergiendo caracterizados los distintos usos dados a los conocimientos matemáticos que, a su vez, son los indicadores de la comprensión y competencia matemáticas que Nadia despliega en esta ocasión.

Por otra parte, también hemos elegido de manera intencionada esta tarea matemática por ser escolar, convencional, descontextualizada, pura, abstracta y sin utilidad práctica aparente. La comprensión y competencia matemáticas también se despliegan en la resolución de este tipo de situaciones (González, 2008; Niss y Højgaard, 2011). En definitiva, nuestro propósito es el de evidenciar la comprensión y competencia de Nadia a través de un proceso de interpretación que busca delimitar y caracterizar los distintos usos dados a los conocimientos matemáticos en dicha tarea.

\section{Rastros de comprensión en la actividad matemática de Nadia}

El registro escrito elaborado por Nadia pone de manifiesto una estrategia de resolución de la tarea en la que intervienen distintos conocimientos matemáticos utilizados de forma relacionada. De acuerdo con nuestros planteamientos, la interpretación de la comprensión y competencia matemáticas que Nadia despliega en este episodio requiere, en primer lugar, identificar y delimitar entre todo lo registrado de su actividad matemática las trazas o rastros de comprensión que pueden considerarse indicadores de algún uso dado al conocimiento matemático. En esta fase pretendemos describir los vínculos establecidos entre las situaciones problemáticas específicas a las que se va enfrentando a lo largo del proceso de resolución y los conocimientos matemáticos puestos en juego para superarlas. Nadia comienza la resolución del siguiente modo:

1 Son las 14:20 h del domingo... [5 de febrero de 2012]. Estoy interpretando el problema y creo que es más fácil de lo que parece.

$25(2 x-\Delta)-(\nabla x+3)(x-2)=0$

3 Entonces, si $x=1$, lo interpretariamos asi: $5(2-\Delta)-(\nabla \cdot 1+3) \cdot(1-2)=0$

$4 \quad$ Creo que manteniendo el resultado 0 desde el inicio de la ecuación podría resultar.

$5 \quad 5(2-2)=5 \cdot 0=0$

6 Entonces: $0-(\nabla \cdot 1+3) \cdot(1-2)=0$

$70-$ un número más tres por $-1=0$. He probado con varios números, pero creo que ese método no es adecuado.

Su interpretación del enunciado la lleva, en primer lugar, a sustituir en la expresión algebraica la incógnita $x$ por el valor 1 (línea 3). A continuación, en la nueva expresión algebraica obtenida tras la sustitución percibe una resta. Como estrategia para mantener el resultado final 0 , parece aplicar la propiedad 0 - $0=0$ o emplear el argumento de si todo fuera 0 , el resultado sería 0 . Lo hace intentando encontrar valores para $\Delta$ y $\nabla$ que hagan 0 tanto al minuendo como al sustraendo. Consigue hacer 0 al minuendo (primera parte de la ecuación) tomando $\Delta=2$ (5). Pero al probar con algunos otros números no encuentra ningún valor adecuado para $\nabla$ que permita anular al sustraendo (segunda parte de la ecuación). Esta circunstancia la lleva a considerar inadecuada la estrategia de la «anulación» (7). Por otra parte, el carácter de la notación algebraica mostrado en esta línea, en la que se mezclan sistemas de representación verbal y simbólica, también evidencia una cierta disposición de la alumna al empleo de versiones poco convencionales del lenguaje matemático. En definitiva, en esta primera fase de la resolución ya se muestran indicios de modelización matemática elemental, al sustituir la variable por un valor dado y encontrar una nueva expresión algebraica. También indicadores de competencia 
en resolución de problemas así como en pensamiento y razonamiento matemático en el uso de estrategias matemáticas como la de ensayo y error (aunque sobre una suposición falsa). Además, es clara la pertenencia de Nadia a su cultura matemática escolar a través del uso no estándar dado al lenguaje matemático. La resolución de la tarea prosigue con la consideración de una variante de la estrategia de «anulación»:

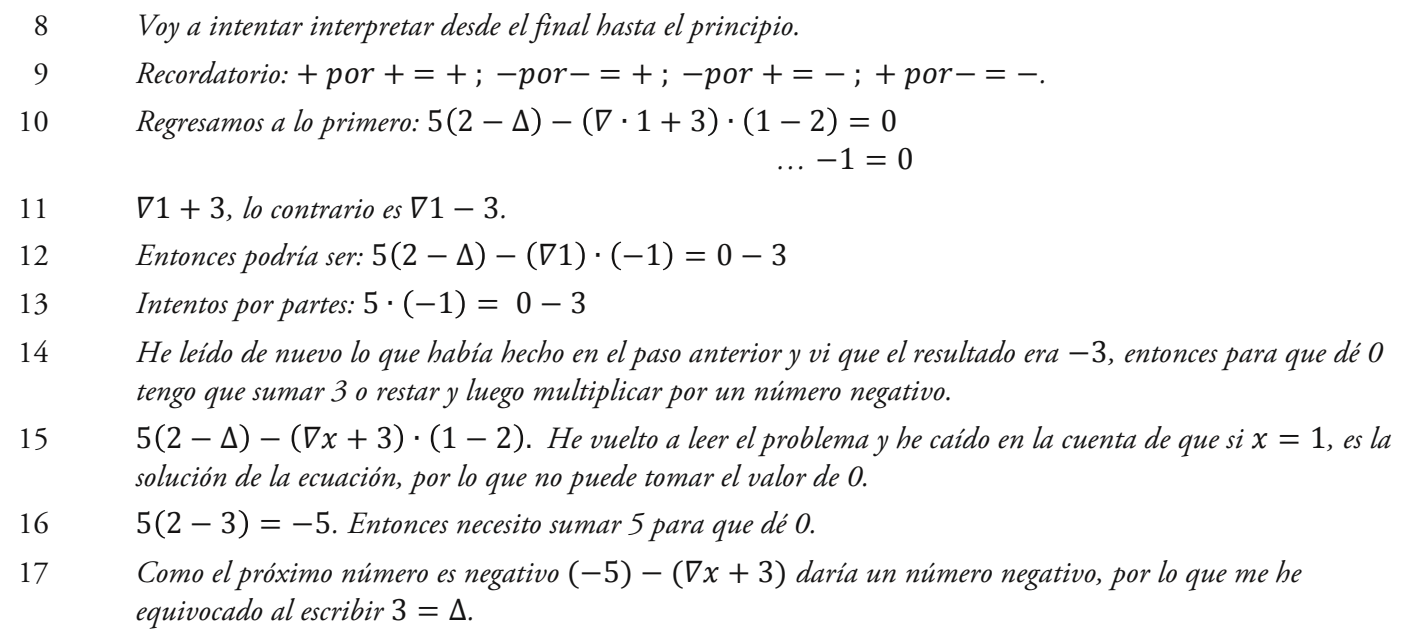

En esta fase de la resolución, Nadia se recuerda la «regla de los signos» para la multiplicación de números enteros (9) con la intención de aplicarla a continuación. Por otro lado, vuelve a emplear una versión poco convencional de escritura matemática, esta vez con la introducción de los puntos suspensivos como táctica para evitar escribir parcialmente la misma expresión algebraica por segunda vez (10). Ahora la estrategia consiste en reescribir la expresión algebraica inicial, haciendo las simplificaciones que ofrece el cálculo aritmético: el signo menos afecta a todo el segundo paréntesis, por lo que el opuesto de +3 es -3 (11) y además la regla de los signos permite concluir que $(-3) \cdot(-1)=+3$. A continuación, traspone +3 del primer miembro al segundo miembro de la ecuación con el correspondiente cambio de signo (12), si bien no podemos afirmar nada acerca de la justificación matemática en la que se apoya para hacer esto. En todo caso, con todo lo anterior consigue reducir o simplificar un poco la expresión algebraica original. A partir de aquí, Nadia da posibles valores a $\Delta, \nabla$ y $x$ (en concreto, prueba con $\Delta=3$ y $x=0$ ) para hacer válida la nueva expresión algebraica, y emplea para ello una estrategia de "compensación» (basada en la propiedad aritmética $\mathrm{a}-\mathrm{a}=0$ ) consistente en lograr 0 mediante la resta de sumandos iguales (13-14). En este punto se da cuenta de que el valor $x=1$ no puede ser modificado (15). Regresa entonces a la expresión algebraica inicial para seguir aplicando en ella su estrategia de "compensación» que le permite renunciar al valor $\Delta=3$ de manera justificada (15-16). En resumen, esta fase se caracteriza sobre todo por el uso instrumental de los conocimientos aritméticos y algebraicos, como la regla de los signos o la simplificación de expresiones algebraicas, junto con estrategias como la revisión de condiciones iniciales. Estos usos son propios de una competencia en resolución de problemas, aunque por ahora la presencia de errores en su aplicación dificulta el desenlace exitoso de la resolución.

Al día siguiente, Nadia retoma la resolución de la tarea recordando algunas propiedades y posibles estrategias útiles: 
Hoy lunes me he querido enfrentar al problema. Teniendo en cuenta el recordatorio de los números positivos y negativos y también recordando que cualquier número multiplicado por 0 da 0 . Entonces puedo intentar obtener $O$ desde el inicio, a ver si resulta.

¡Qué locura! Creo que cada vez me acerco más. ¡Tengo que seguir!

Si el primer número tiene que ser positivo, el $\Delta$ debe ser o 2 o 100 . A lo mejor puede funcionar...

$$
\left.\begin{array}{rl} 
& \rightarrow 2 \\
5(2 x- & \rightarrow 1 \\
& \rightarrow 0
\end{array}\right)
$$

Si fuera 2 seria: $5(2-2)=0$.

$0-(\nabla x+3)(-1)$ iNo funciona! Porque sería 0 - un número positivo por uno negativo, al final sería un número positivo mayor que 0 .

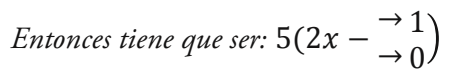

Voy a probar con el 1 para que dé un número positivo.

$26 \quad 5(2 x-1)=5$

$5-(\nabla x+3)(x-2)$

Como luego tengo que por $-1,(x-2)$, tengo que hacer que el número que lo multiplique dé 0 .

$5-5=0 \cdot-1=0$

$\Delta=1$

$\nabla=2$

¡LO LOGRE!

Voy a comprobarlo, para estar al 100\% segura.

$$
\begin{gathered}
5(2-1)-(2+3)(1-2) \\
=5=\quad=-5
\end{gathered}
$$$$
10
$$

$5--5=10$

iiii No!!!!! ¡Me he hecho ilusiones falsas! Bueno, tendré que seguir intentando.

Insiste en el empleo de la estrategia de «compensación». Comienza con la primera parte de la ecuación sugiriendo tres valores posibles para $\Delta$. Prueba primero con $\Delta=2$, pero no consigue cuadrar la segunda parte (20-23). De nuevo, utiliza en la explicación una versión no convencional de lenguaje matemático, en la que lo verbal suple en parte al propio lenguaje algebraico (23). A continuación, prueba con $\Delta=1$ y obtiene 5 para $5 \cdot(2 x-\Delta)(25-26)$. Con el propósito de obtener otro 5 en $(\nabla x+3)(x-2)$ prueba con $\nabla=2$. Al realizar los cálculos no tiene en cuenta la jerarquía en las operaciones (28), por lo que obtiene una solución incorrecta y la sensación de haber resuelto el problema (29-30). Sin embargo, Nadia se muestra cauta en este punto y decide comprobar de nuevo la respuesta dada. Al realizar por segunda vez los cálculos aritméticos derivados de la sustitución de $\Delta, \nabla \mathrm{y} x$, esta vez sin cometer errores, se percata de que en realidad no ha alcanzado el resultado requerido y de que el problema aún permanece abierto (31-33). En esencia, esta fase evidencia indicios del uso del cálculo aritmético, de la comprobación de respuestas y de la modificación de condiciones iniciales, entre otros, que son distintivos de competencia en resolución de problemas, argumentación y pensamiento y razonamiento matemático. Las versiones no convencionales de la notación matemática empleadas hasta ahora también podrían interpretarse como indicadores de una competencia mejorable en modelización matemática. 
La parte final de la resolución transcurre como sigue:

34 A ver, voy a dejar la segunda parte $[(2+3)(1-2)]$ en paz, me voy a poner como meta convertir el primer resultado en negativo $[5(2-1)=5]$. Tiene que ser negativo.

35 Si en $5(2-1)$, estoy multiplicando $5 \cdot 1$, me da positivo, pero si multiplico por -1 me daría negativo. Entonces en lugar de restar $2-1$, sería $2-3$, que es igual a -1 .

Creo que... ;Por fin! $\Delta=3 \nabla=2 ; L O$ LOGRÉ!

Nadia se esfuerza por ajustar y mejorar lo ya realizado. En concreto, mantiene su estrategia de «compensación» así como la segunda parte de la ecuación con el valor $\nabla=2$. Pero ahora modifica la condición inicial impuesta en la línea 20 relativa al carácter positivo de la primera parte de la ecuación (34). Buscando un valor negativo para esta primera parte, decide tomar $\Delta=3$ (35). Tras la correspondiente comprobación final (36-37), Nadia da por resuelta la tarea propuesta.

\section{La competencia matemática de Nadia}

¿Qué puede decirse acerca de la competencia matemática de Nadia a partir de su desempeño en esta tarea? Nuestro modelo de interpretación sugiere transitar desde los rastros de comprensión delimitados durante la actividad matemática, descritos en el apartado anterior, a la caracterización de los usos dados a los conocimientos matemáticos durante la resolución de la tarea. Los usos del conocimiento matemático son los que dan cuenta de la comprensión de Nadia y se proponen como referencia objetiva con la que interpretar su competencia matemática. Es decir, nos servimos de estos usos para caracterizar el perfil de competencia matemática que Nadia despliega en su intento por resolver la situación concreta que se le plantea en esta ocasión. Estos usos son los que definen finalmente la comprensión y competencia matemáticas que Nadia pone en juego durante el episodio. La tabla 1 recoge las evidencias más destacadas de tales usos, organizadas de acuerdo a su pertinencia.

Tabla 1.

Evidencias de los usos dados al conocimiento matemático

\begin{tabular}{ll}
\hline \multicolumn{1}{c}{ Línea } & \multicolumn{1}{c}{ Usos del conocimiento matemático } \\
\hline $1-3$ & Pertinentes \\
3 & Evterpretación matemática de la tarea (propósito, datos, etcétera) \\
4 & Aplicación de la estrategia de «anulación» (propiedad $0-0=0)$ \\
$5-7,13,16,22,28$, & Cálculos aritméticos \\
$32,34-37$ & \\
9 & Regla de los signos para la multiplicación de números enteros \\
$11-12$ & Manipulación de ecuaciones y simplificación de expresiones algebraicas \\
$13-14,20-23$ & Aplicación de la estrategia de «compensación» (propiedad $a-a=0)$ \\
15 & Revisión y corrección de condiciones fijas (de $x=0$ a $x=1)$ \\
$31-33,36-37$ & Comprobación de respuestas propias \\
34 & Flexibilidad en la modificación de condiciones iniciales \\
\hline
\end{tabular}




\begin{tabular}{ll}
\hline \multicolumn{1}{c}{ Línea } & \multicolumn{1}{c}{ Usos del conocimiento matemático } \\
\hline $7,10,23$ & Alterados \\
$13-14$ & Variantes en la notación matemática: prácticas escolares propias \\
20 & Modificación de condiciones necesariamente fijas (de $x=1$ a $x=0)$ \\
28 & Supuestos infundados sobre el signo de las partes de la ecuación \\
\hline & Jerarquía de las operaciones mal aplicada \\
$10-12$ & Inciertos \\
\hline
\end{tabular}

Los usos que la estudiante da a los conocimientos matemáticos pueden considerarse pertinentes si, adoptando alguna de sus formas posibles de su dimensión fenómeno-epistemológica, contribuyen a la resolución de la tarea. De manera más precisa, Nadia se va enfrentando en distintos momentos del proceso de resolución a situaciones y problemas específicos que son propios del ámbito fenómenoepistemológico de determinados conocimientos matemáticos. Las evidencias de un uso pertinente emergen cuando ella identifica tales conocimientos como organizadores de esas situaciones intermedias y finalmente decide emplearlos, en alguna de sus variantes legítimas, como medios para su resolución. Entre las evidencias destacadas que respaldan el hecho de que Nadia utiliza de forma pertinente diversos conocimientos matemáticos particulares durante la resolución de la tarea se encuentran las siguientes: (a) manifestar destreza en los cálculos aritméticos básicos; (b) aplicar procedimientos algebraicos como la evaluación numérica de una incógnita o la manipulación de ecuaciones, y $(c)$ aplicar propiedades numéricas y algebraicas como la regla de los signos para la multiplicación de números enteros o las estrategias de «anulación» y "compensación».

Por otra parte, en la resolución de problemas el uso de conocimientos matemáticos específicos como los ya mencionados puede llevar aparejada la aplicación de heurísticos que actúan como mecanismos de gestión y control del proceso de resolución y que, no siendo exclusivos, son propios y característicos del quehacer matemático, tales como la comprobación de resultados, la revisión de procedimientos o la modificación de estrategias. El uso pertinente de este tipo de conocimiento también es considerado desde nuestro enfoque como indicador de comprensión y competencia matemáticas. Nadia da muestras positivas de su competencia a través de esta faceta al interpretar el enunciado de la tarea, revisar y corregir condiciones inicialmente fijadas para la incógnita $x$, y comprobar respuestas propias.

Todos estos conocimientos mencionados, matemáticos o no, puestos en acción por Nadia le permiten finalmente alcanzar la solución correcta al problema planteado. Desde nuestra perspectiva, los interpretamos como evidencias positivas que dan cuenta o caracterizan de un modo favorable la comprensión y competencia matemáticas de Nadia. En concreto, los usos pertinentes evidenciados revelan en esta ocasión competencias matemáticas específicas en modelización, resolución de problemas, pensamiento y razonamiento, argumentación y comunicación.

Ahora bien, al mismo tiempo es preciso reconocer que durante el episodio descrito la estudiante también pone en juego conocimientos matemáticos empleados de forma no pertinente o inapropiada. Son usos alterados del conocimiento matemático. Ello sucede fundamentalmente como consecuencia de no haber establecido en ciertos momentos los vínculos situación-conocimiento necesarios para progresar en la resolución. En ocasiones, Nadia no llega a identificar algunas situaciones particulares como susceptibles de poderse resolver mediante la aplicación de los correspondientes conocimientos matemáticos que son sus medios de organización (Puig, 2006). En su lugar, pone en acción alteraciones de esos conocimientos matemáticos requeridos, que llegan a perjudicar y entorpecer sus avances en la resolución de la tarea. Nos referimos, entre otros ejemplos, a su decisión de modificar condiciones fijas, a las suposiciones falsas hechas sobre el signo de algunas partes de la ecuación estudiada o a la aplica- 
ción errónea de la jerarquía de las operaciones. Estas variantes en el uso del conocimiento matemático también contribuyen a la caracterización de la competencia matemática de Nadia, interpretándolas como evidencias desfavorables de esta. Los usos alterados evidenciados aquí son indicadores de defectos ocasionales en modelización, pensamiento y razonamiento matemático y resolución de problemas, entre otras competencias matemáticas específicas.

El empleo ocasional que la alumna hace de versiones no convencionales para la notación algebraica, que también consideramos un uso alterado del conocimiento matemático, merece una atención especial por nuestra parte. En principio, la simplificación y economía en la escritura que este uso aporta parece que contribuye favorablemente a la resolución de la tarea durante el episodio. Por esta razón, podemos contemplarlo como una evidencia positiva más de la competencia matemática de Nadia. Ahora bien, al mismo tiempo estas versiones parecen ser consecuencia de unas prácticas matemáticas escolares en las que un tipo particular de uso del conocimiento matemático resulta cotidiano. Esto lo interpretamos como una manifestación de que la situación particular de la competencia está influenciada en algunos aspectos por el entorno escolar propio de los alumnos y por las prácticas matemáticas particulares en las que se ven inmersos; prácticas que para ser correctas no podrán desviarse en exceso de las consideradas usuales.

Finalmente, junto con los usos pertinentes y alterados del conocimiento matemático hemos de admitir la presencia de usos inciertos durante la resolución, de los que no podemos extraer evidencias claras acerca de la comprensión y la competencia desplegadas. Aceptamos esta circunstancia no como una limitación metodológica de nuestro modelo interpretativo sino más bien como una particularidad de la interpretación en cualquiera de sus variantes (Gallardo, González y Quintanilla, 2014). Es el caso, por ejemplo, de la justificación que pudiera dar Nadia de la transposición de términos en la ecuación algebraica, desconocida para nosotros en este episodio concreto.

\section{CONCLUSIÓN}

Consideramos que las controversias delimitadas y discutidas en este estudio en torno a la descripción por competencias del currículo de matemáticas merecen una mayor atención y reflexión por parte de los diseñadores curriculares, investigadores y profesores a la hora de planificar y aplicar el currículo, trabajar en el aula de matemáticas y/o evaluar los logros de los estudiantes en términos de competencias. Como contribución específica al debate, nuestro enfoque interpretativo intenta integrar la competencia y el conocimiento matemático dentro del mismo proceso complejo de la comprensión. En este trabajo hemos pretendido mostrar cómo el fomento de las capacidades y destrezas en el uso de los objetos matemáticos básicos en tareas problemáticas planificadas y concretas, característico del currículo descrito por competencias, resulta compatible con el estudio de las diversas variantes fenómeno-epistemológicas del conocimiento matemático. La dimensión fenómeno-epistemológica de nuestro enfoque interpretativo propone unos referentes teórico-metodológicos operativos sobre el conocimiento matemático y sobre la comprensión en matemáticas y su valoración. Consideramos que ello puede resultar eficaz en la interpretación de la competencia en matemáticas.

La comprensión del conocimiento matemático y su principal consecuencia, la competencia matemática, quedan entonces caracterizadas desde nuestros planteamientos por el conjunto de evidencias recabadas acerca de los usos dados a los conocimientos matemáticos que emergen de la actividad matemática desplegada en el intento por resolver las tareas. Estas evidencias provienen de los propios conocimientos matemáticos, son inherentes a ellos y a las acciones particulares realizadas con ellos. Finalmente, constituyen la referencia interna objetiva desde la que proponemos interpretar la competencia matemática en cada caso. Además, la interacción en las distintas aulas particulares y la toma de conciencia de la actividad matemática que en ellas se desarrolla configuran el escenario de interpreta- 
ciones y acciones mediadas por el contexto social y cultural en el que se configuran y desarrollan las competencias matemáticas de los alumnos. En nuestra opinión, se trata de una alternativa complementaria a la opción curricular vigente de sustentar la valoración de la competencia matemática en términos de listas genéricas de atributos externos definidos con independencia de la propia actividad matemática intrínseca de cada estudiante.

Finalmente, a través del episodio descrito, hemos querido mostrar en la práctica la posibilidad y la complejidad de interpretar la comprensión y la competencia en tareas puramente matemáticas, en apariencia alejadas del ámbito de la utilidad práctica de las matemáticas y del uso cotidiano del conocimiento matemático más característico del marco de descripción por competencias del currículo. Pensamos que este tipo de tareas también pertenecen al ámbito de la funcionalidad de las matemáticas y que el desempeño de los estudiantes frente a ellas genera nuevas oportunidades para evidenciar y desarrollar comprensión y competencia en matemáticas. A nuestro entender, no reconocer esto supondría relegar las competencias matemáticas a un lugar que no les corresponde.

\section{REFERENCIAS BIBLIOGRÁFICAS}

Álvarez, M.a D., Hernández, J., Miranda, A.Y., Moreno, M.ª., Parra, S., Redondo, M., Redondo, R., Sánchez, M. a'T., Santos, T. y Serrano, E. (2008). Matemáticas 2. ${ }^{\circ}$ ESO. Andalucía. Madrid: Grazalema Santillana.

Brown, T. (1996). Towards a hermeneutical understanding of mathematics and mathematical learning. En P. Ernest (Ed.). Constructing mathematical knowledge: Epistemology mathematical education. London: Routledge Falmer, pp. 141-150.

http://dx.doi.org/10.1007/978-94-010-0726-9

Brown, T. (2001). Mathematics education and language. Interpreting hermeneutics and post-structuralism. Dordrecht, The Netherlands: Kluwer Academic Publishers.

Brown, T. (2008, octubre). Making mathematics inclusive: interpreting the meaning of classroom activity. Philosophy of Mathematics Education Journal, 23. Disponible en línea: <http://people.exeter. ac.uk/PErnest/pome23/index.htm>.

Duval, R. (2006). A cognitive analysis of problems of comprehension in a learning of mathematics. Educational Studies in Mathematics, 61(1/2), pp. 103-131. http://dx.doi.org/10.1007/s10649-006-0400-z

Font, V., Godino, J.D. y Gallardo, J. (2013). The emergence of objects from mathematical practices. Educational Studies in Mathematics, 82(1), pp. 97-124. http://dx.doi.org/10.1007/s10649-012-9411-0

Gallardo, J. y González, J.L. (2006). Assessing understanding in mathematics: steps towards an operative model. For the Learning of Mathematics, 26(2), pp. 10-15.

Gallardo, J., González, J.L. y Quispe, W. (2008a). Interpretando la comprensión matemática en escenarios básicos de valoración. Un estudio sobre las interferencias en el uso de los significados de la fracción. Revista Latinoamericana de Investigación en Matemática Educativa RELIME, 11(3), pp. 355-382.

Gallardo, J., González, J.L. y Quispe, W. (2008b). Rastros de comprensión en la acción matemática. La dimensión hermenéutica de un modelo operativo para la interpretación en matemáticas. En R. Luengo, B. Gómez, M. Camacho y L.J. Blanco (Eds.) Actas del XII Simposio de la Sociedad Española de Investigación en Educación Matemática SEIEM (pp. 283-293). Badajoz: Sociedad Extremeña de Educación Matemática- SEIEM. 
Gallardo, J., González, J.L. y Quintanilla, V.A. (2013). Tareas, textos y usos del conocimiento matemático: aportes a la interpretación de la comprensión desde el cálculo aritmético elemental. Educación Matemática, 25(2), pp. 61-88.

Gallardo, J., González, J.L. y Quintanilla, V.A. (2014). Revisiting understanding in mathematics. Quadrante, 23(1), pp. 63-78.

Godino, J.D. (2002a). Competencia y comprensión en matemáticas: ¿qué son y cómo se consiguen? UNO, 29, pp. 9-19.

Godino, J.D. (2002b). Perspectiva semiótica de la competencia y comprensión matemática. La matematica e la sua didattica, 4, pp. 434-450.

GonzÁlez, J.L. (2008). Competencias básicas y competencias matemáticas específicas. Disponible en línea: <http://www.gonzalezmari.es>.

Gresalfi, M., Martin, T., Hand, V. y Greeno, J. (2009). Constructing competence: an analysis of student participation in the activity systems of mathematics classrooms. Educational Studies in Mathematics, 70(1), pp. 49-70.

http://dx.doi.org/10.1007/s10649-008-9141-5

Habermas, J. (1999). Teoría de la acción comunicativa I. Madrid, España: Taurus.

Llewellyn, A. (2012). Unpacking understanding: the (re)search for the Holy Grail of mathematics education. Educational Studies in Mathematics, 81(3), pp. 385-399. http://dx.doi.org/10.1007/s10649-012-9409-7

LupiáñEZ, J.L. y Rico, L. (2006). Análisis didáctico y formación inicial de profesores: competencias y capacidades en el aprendizaje de los escolares. En P. Bolea, M.a. González y M. Moreno (Eds.) Actas del X Simposio de la Sociedad Española de Investigación en Educación Matemática SEIEM. Huesca: Instituto de Estudios Altoaragoneses-Universidad de Zaragoza, pp. 225-236.

MeEL, D.E. (2003). Modelos y teorías de la comprensión matemática: Comparación de los modelos de Pirie y Kieren sobre la evolución de la comprensión matemática y la teoría APOE. Revista Latinoamericana de Investigación en Matemática Educativa RELIME, 6(3), pp. 221-278.

National Council of Teachers of Mathematics (2000). Principles and standards for school mathematics. Reston, VA: Author.

Niss, M. (Ed.) (2002). Mathematical competencies and the learning of mathematics: The Danish KOM Project. Roskilde, Denmark: Roskilde University.

Niss M. y HøjGAARD, T. (Eds.) (2011). Competencies and mathematical learning. Ideas and inspiration for the development of mathematics teaching and learning in Denmark. Roskilde, Denmark: IMFUFA.

OCDE (2003). The PISA 2003 Assessment Framework. Mathematics, Reading, Science and Problem Solving Knowledge and Skills. Paris: Author.

Popper, K. (2005). El mito del marco común. Barcelona: Paidós.

PuIG, L. (2006). Sentido y elaboración del componente de competencia de los modelos teóricos locales en la investigación de la enseñanza y aprendizaje de contenidos matemáticos. En P. Bolea, M.aJ. González y M. Moreno (Eds.). Actas del X Simposio de la Sociedad Española de Investigación en Educación Matemática SEIEM. Huesca: Instituto de Estudios Altoaragoneses-Universidad de Zaragoza, pp. 107-126.

Rico, L. y Lupiáñez, J.L. (2008). Competencias matemáticas desde una perspectiva curricular. Madrid: Alianza Editorial.

Ricceur, P. (2002). Del texto a la acción. México DF: Fondo de Cultura Económica.

SierpinsKa, A. (2000). Mathematics classrooms that promote understanding [Review of the book Mathematics classrooms that promote understanding]. ZDM-The International Journal on Mathematics Education, 2, pp. 45-50.

UNESCO (2012). Challenges in basic mathematics education. Paris: Author. 


\title{
On the assessment of mathematical competence: keys in moving towards an interpretive approach
}

\author{
Jesús Gallardo Romero, José Luis González Marí,Verónica Aurora Quintanilla Batallanos \\ Universidad de Málaga \\ gallardoromero@telefonica.net, gmari@uma.es, veronicaquintanilla@uma.es
}

At present, the development of mathematical competence is presented in the basic education curriculum as a priority curricular objective. The curriculum seeks to promote mainly which students know what to do with mathematics, to use the mathematical knowledge learned in school in order to act in common situations of everyday life.

Recently several controversies have emerged which suggest that some of the principles of the current curriculum framework by competency are to be revised and refined. These refer to the relationship of mathematical competence with the phenomenon of understanding, with the mathematical knowledge itself, with the tasks and mathematical situations and the interpretation of mathematical activity in the classroom. As a contribution to the debate raised, we propose to move towards a more interpretive approach to mathematical competence.

First, we relate the mathematical competence in a functional point of view of understanding through the use of mathematical knowledge. Understanding in mathematics makes the learner competent for developing the appropriate and contextualized responses involving the recordable and interpretable use, if any, of mathematical knowledge in any of the categories and possible ways of its phenomenon-epistemological dimension.

Second, the phenomenological and epistemological complexity of the mathematical knowledge takes centre stage in the development of mathematical competence. Based on this, we include a proposed phenomenological and epistemological analysis of mathematical knowledge that gives us an objective reference from which to support the understanding and interpretation of the mathematical competence.

Third, mathematical competence is certainly linked to the interpretation of mathematical actions of students in the classroom. In our case, we added to the process of interpretation an interpretive cycle that corresponds to the problematic transition between mathematical activity, on the one hand, and understanding and mathematical competence, on the other. The interpretation addresses the externalization and characterization of the uses of mathematical knowledge arising from traces of emerging understanding from the students' own records of mathematical activity.

As evidence of the operation of our proposal, in the practice we address the interpretation of the mathematical understanding and competence of a second year high school student in the course of solving an algebraic task. The interpretation scene on this occasion is the written protocol prepared for her to try to solve a problem that requires the combined and related use of varied mathematical knowledge specific to both the arithmetic and algebraic areas, and the use of various strategies of management and control of the process of solving mathematical work itself. In this episode we illustrate the phenomenon-epistemological links which the student establishes, and how she establishes them, amongst situations that appear during the resolution and the possible mathematical knowledge used as a means to solve them. In the first phase, we identify and set amongst everything recorded of her mathematical activity the trails or traces of understanding that can be considered indicators of some use made of the mathematical knowledge. In a second phase, we make use of these relevant or altered uses, in order to characterize the mathematical competence profile which the student displays in the example.

As main conclusions, our interpretive approach allows us to manage a more inclusive position from the inherent complexity to the interpretation of mathematical competence, integrating competence and mathematical knowledge within the complex process of understanding. Furthermore, we show how the fostering of abilities and skills in the use of basic mathematical objects in planned and specific problematic tasks, characteristic of the curriculum by competency, is compatible with the study of various phenomena-epistemological variants of mathematical knowledge. Furthermore, we show that the collected evidence on emerging uses of mathematical activity, with which we characterize the understanding, come from mathematical knowledge itself, are inherent to them and the particular actions performed with them. Therefore, they consist of an internal objective reference, complementary to the existing curricular option of supporting the assessment of mathematical competence in terms of generic lists of external attributes defined independently of the intrinsic mathematical activity of each student. Finally, we also show the possibility of interpreting the understanding and competence in purely mathematical tasks, seemingly far removed from the scope of both the practical use of mathematics and everyday use of mathematical knowledge, most characteristic of the current curriculum by competency. 